\title{
A Novel Mouse Model of Staphylococcus aureus Chronic Osteomyelitis That Closely Mimics the Human Infection
}

\section{An Integrated View of Disease Pathogenesis}

\author{
Sarah A. Horst, ${ }^{*}$ Verena Hoerr, ${ }^{\dagger}$ \\ Andreas Beineke, ${ }^{\ddagger}$ Carolin Kreis, ${ }^{\S}$ \\ Lorena Tuchscherr, " Julia Kalinka," \\ Sabine Lehne, ${ }^{*}$ Ina Schleicher," Gabriele Köhler, ${ }^{* *}$ \\ Thomas Fuchs, ${ }^{\S}$ Michael J. Raschke, ${ }^{\S}$ \\ Manfred Rohde," Georg Peters, " Cornelius Faber, ${ }^{\dagger}$ \\ Bettina Löffler," and Eva Medina* \\ From the Infection Immunology Research Group," and the \\ Department of Medical Microbiology," Helmboltz Centre for \\ Infection Research, Braunschweig; the Department of Clinical \\ Radiology, ${ }^{\dagger}$ the Clinic of Trauma-, Hand-, and Reconstructive \\ Surgery, ${ }^{\S}$ and the Gerhard-Domagk-Institute of Pathology, ${ }^{* *}$ \\ University Hospital of Münster, Albert-Schweitzer Campus, \\ Münster; the Institute for Pathology, University of Veterinary \\ Medicine Hannover, Hannover; and the Institute of Medical \\ Microbiology, "Iniversity Hospital of Münster, Münster, Germany
}

Osteomyelitis is a serious bone infection typically caused by Stapbylococcus aureus. The pathogenesis of osteomyelitis remains poorly understood, mainly for lack of experimental models that closely mimic human disease. We describe a novel murine model of metastatic chronic osteomyelitis initiated after intravenous inoculation of $S$. aureus microorganisms. The bacteria entered bones through the bloodstream and, after an acute phase with progressive growth (first 2 weeks after infection), they remained at constant numbers for up to 56 days (chronic phase). Clinical signs of illness and systemic inflammation were apparent only during the acute phase. Bone destruction and remodeling processes were readily detectable by magnetic resonance and $X$-ray imaging 3 weeks after infection, and high levels of bone deformation were observed during the chronic phase. Histological examination of infected bones demonstrated suppurative inflammation with foci of intense bacterial multiplication and necrosis during acute infection and osteoclastic resorption accompanied by new woven bone formation during chronic infection. Transmission electron microscopy revealed $S$. aureus microorganisms forming microcolonies within the nonmineralized collagen matrix or located intracellularly within neutrophils. In summary, our mouse model of staphylococcal hematogenous osteomyelitis precisely reproduces most features of the human disease. Although the extent of lesions in the chronic phase was subject to variation, this model is ideal for testing and monitoring novel treatment modalities via noninvasive imaging. (Am J Pathol 2012, 181:1206-1214; http://dx. doi.org/10.1016/j.ajpath.2012.07.005)

Osteomyelitis is an infection of the bone that can be associated with high levels of inflammation and bone tissue destruction. Staphylococcus aureus is the predominant causative agent of osteomyelitis worldwide. ${ }^{1}$ The infection is painful and debilitating, and often takes a chronic course that is extremely difficult to treat. Consequently, the rate of clinical failures in osteomyelitis treatment is high, and frequently results in loss of function and/or amputation. ${ }^{2}$ Although the increasing spread of antibiotic-resistant strains such as methicillin-resistant $S$.

Supported in part by the Federal Ministry of Education and Research [Bundesministerium für Bildung und Forschung-BMBF "Medizinische Infektionsgenomik" (0315830B and 01KI1009B)], by the German Research Foundation (SFB TR34, C12, and Z3), and by the Interdisciplinary Center for Clinical Research-Münster [Interdisziplinäres Zentrum für Klinische Forschung-IZKF Münster (Löf2/030/10 and SAMRI)].

Accepted for publication July 2, 2012.

B.L. and E.M. contributed equally to this work.

Supplemental material for this article can be found at http://ajp. amjpathol.org or at http://dx.doi.org/10.1016/j.ajpath.2012.07.005.

Address reprint requests to Eva Medina, Ph.D., Infection Immunology Research Group, Helmholtz Centre for Infection Research, Inhoffenstraße 7, 38124 Braunschweig, Germany. E-mail: eva.medina@helmholtz-hzi.de. 
aureus (MRSA) $)^{3}$ may be responsible, at least in part, for treatment failure, many therapy-refractory osteomyelitis infections are caused by strains that have not acquired resistance to antibiotics. ${ }^{4,5}$ Therefore, both the complex interaction of staphylococci with osseous tissue and the wide array of strategies used by S. aureus to circumvent the host immune system are most likely the major determinants for ineffectiveness of antimicrobial treatment. ${ }^{6,7}$

In general, osteomyelitis can be categorized into three groups, according to the route used by the infecting bacterium to gain access to the bone. ${ }^{2}$ First, in hematogenous osteomyelitis, staphylococci access the bone tissue via the bloodstream. This form of osteomyelitis affects predominantly prepubertal children and elderly patients. Little is known about the bacterial factors involved in bone tropism, and even less about the host predisposing factors. Treatment is particularly complicated when the bone infection has reached a chronic stage, which can be associated with either necrosis of epiphysis, limb length discrepancy, or pathological fracture. ${ }^{8,9}$ Second, osteomyelitis can also develop by way of a contiguous spread from a local infection after trauma, bone surgery, or joint replacement. As the number of joint replacements has dramatically risen in the last decades, the infections associated with these procedures have also increased. Finally, osteomyelitis can be secondary to peripheral vascular insufficiency. This form of osteomyelitis occurs predominantly in diabetic patients and usually originates from an infected foot ulcer that spreads to the bone. This type of osteomyelitis affects a large percentage of the 200 million patients with diabetes worldwide and requires prolonged and repeated hospital admissions. ${ }^{10,11}$ In all its forms, osteomyelitis is the most frequent cause of nontraumatic limb amputation, because antimicrobial compounds often fail to clear the infection.

Although the route of bacterial inoculation into bone tissue differs among the various types of osteomyelitis, all forms of osteomyelitis share common features, including the development of distinct sequential clinical stages depending on the duration of infection (ie, acute and chronic osteomyelitis). ${ }^{2,12}$ Acute osteomyelitis develops within the first days or weeks after onset of infection and is usually associated with intense inflammation, infiltration of polymorphonuclear neutrophils, and systemic symptoms. During this stage, the vascular supply to the bone is decreased, leading to large areas of dead bone., ${ }^{2,13}$ If acute osteomyelitis is inadequately treated, it can become chronic, leading to specific pathological symptoms, including abscess formation, remodeling of bone, increased bone fragility, damaged vasculature, accelerated bone necrosis, and formation of sequestra. ${ }^{14,15}$ Chronic osteomyelitis remains challenging and difficult to treat. Furthermore, relapses of the disease and late activation several decades after the acute episode have been reported. ${ }^{16,17}$

A better understanding of the complex pathogenesis of osteomyelitis, as well as a better definition of the mechanisms used by $S$. aureus microorganisms to establish infection in the bone, are required for the development of more effective therapeutic modalities. To gain this understanding, we have developed a novel murine model of hematogenous S. aureus osteomyelitis that closely mimics the pathological features of both acute and chronic human disease. In this experimental model, C57BL/6 mice are intravenously infected with $S$. aureus microorganisms, and osteomyelitis is generated after hematogenous bacterial seeding without additional trauma, tissue manipulation, or incorporation of foreign bodies. Major hallmarks of acute and chronic osteomyelitis were confirmed by radiographical imaging, magnetic resonance imaging (MRI), histopathology evaluation, and electron microscopy. This murine model represents a robust platform for reliably investigating the bacterial and host factors involved in establishment and control of chronic $S$. aureus osteomyelitis. The model may also prove suitable for assessment of preclinical studies for testing and optimizing novel therapeutic treatments.

\section{Materials and Methods}

\section{Bacterial Strain and Inoculum}

S. aureus strain 6850 (53657; ATCC, Manassas, VA) ${ }^{18}$ was cultivated overnight in brain-heart infusion medium under shaking conditions at $37^{\circ} \mathrm{C}$. Bacteria were washed twice with sterile PBS, the bacterial suspension was adjusted to optical density at $600 \mathrm{~nm}\left(\mathrm{OD}_{600}=1\right)$ and stored at $-80^{\circ} \mathrm{C}$ until use. S. aureus strain 6850 exhibits resistance to penicillin $\mathrm{G}$ [minimum inhibitory concentration $(\mathrm{MIC})>0.5<1$ ] and ampicillin (MIC $>4<8$ ) and is fully susceptible to flucloxacillin, cephalosporins, rifampicin, clindamycin, and vancomycin.

\section{Mice and Infection Model}

Pathogen-free C57BL/6 10-week-old female mice were obtained from Harlan-Winkelmann (Borchen, Germany). The animals were maintained according to institutional guidelines in individually ventilated cages and were given food and water ad libitum. All experiments were approved by the local ethical board [Lower Saxony State Office for Consumer Protection and Food Safety (Niedersächsisches Landesamt für Verbraucherschutz und Lebensmittelsicherheit-LAVES) and the North Rhine-Westphalia Agency for Nature, Environment, and Consumer Protection (Landesamt für Natur, Umwelt und Verbraucherschutz NordrheinWestfalen-LANUV) (permit no. 33.9-42502-04-10/0296)].

Mice were inoculated with $10^{6}, 5 \times 10^{5}$, or $2 \times 10^{5} \mathrm{CFUs}$ of $S$. aureus in $150 \mu \mathrm{L}$ of PBS via a lateral tail vein. Mice were sacrificed by $\mathrm{CO}_{2}$ asphyxiation at the indicated time of infection. For enumeration of bacteria in the tibiae and kidneys of infected mice, homogenates were prepared in PBS and plated in 10-fold serial dilutions on blood agar.

\section{ELISA}

Serum levels of IL-6 were determined by enzyme-linked immunosorbent assay (ELISA) according to the manufacturer's recommendations (BD Biosciences, San Diego, CA). 


\section{Histology}

Tibial bones were removed and fixed in 10\% formalin, decalcified in 10\% EDTA solution for 48 hours, and then embedded in paraffin. Tissue slides of bone samples ( $3 \mu \mathrm{m}$ thick) were stained with H\&E and examined under light microscopy.

\section{Transmission Electron Microscopy}

Infected tibiae were fixed with 2\% glutaraldehyde/5\% formaldehyde in cacodylate buffer for 1 hour on ice, washed with buffer, and osmificated with $1 \%$ aqueous osmium for 1 hour at room temperature. Samples were then dehydrated with a graded series of acetone and embedded in Spurr's epoxy resin according to procedures described previously. ${ }^{19}$ Ultrathin sections were cut with a diamond knife, picked up with Butvar polyvinyl butyral resin-coated grids, counterstained with uranyl acetate and lead citrate, and examined under a Zeiss TEM910 transmission electron microscope (Carl Zeiss, Oberkochen, Germany) at an acceleration voltage of 80 $\mathrm{kV}$. Images were recorded digitally at calibrated magnifications using a Slow-Scan CCD camera $(1024 \times 1024$ pixels; ProScan, Scheuring, Germany) with iTEM-Software (Olympus Soft Imaging Solutions, Münster, Germany). Contrast and brightness were adjusted with Adobe Photoshop CS3.

\section{MRI and X-Ray Radiography}

In vivo MRI was performed in mice before infection and at days $3,7,14,22,35,49$, and 63 after bacterial inoculation. Images were acquired at 9.4 T on a BioSpec 94/20 system (Bruker, Ettlingen, Germany) equipped with a 0.7 $\mathrm{T} / \mathrm{m}$ gradient system. Lesions and bone structure were imaged by a three-dimensional fast low-angle shot $(\mathrm{FLASH})$ sequence with the following parameters: matrix size, $512 \times 256 \times 102$; field of view, $4.5 \times 2.5 \times 2.0 \mathrm{~cm}^{3}$; TR/ TE, $20.0 \mathrm{~ms} / 3.1 \mathrm{~ms}$; and flip angle (FA), 10 degrees. During MRI, animals were anesthetized with $2 \%$ isoflurane and were monitored for core body temperature and respiration rate using a MRI-compatible monitoring system (SA Instruments, Stony Brook, NY). To reduce motion artifacts, scans were acquired with respiratory triggering. In magnetic resonance images, inflamed regions (defined as areas of inflammation with edema formation that appear bright in the MRI images) and tibia volume were segmented using Amira software version 5.4.0 (Visage Imaging, Berlin, Germany).

In addition to MRI, whole-body X-ray radiography was performed on a Kodak imaging system using an exposure time of 40 seconds.

\section{Biomechanical Measurement Using Torsion and Loading Test}

Tibiae removed from uninfected and S. aureus-infected mice were prepared by removing the periosteum and by fixing the distal and the proximal end in a metal fixture using polymethylmethacrylate bone cement (PMMA;
Heraeus Medical, Wehrheim, Germany). After the cement had hardened, all tibiae were placed in the fixture. The torsional bending test was conducted using a torsional testing machine (LR5K-plus; Lloyd Instruments, Bognor Regis, UK) connected to a speed controller and a data acquisition system. Torsional testing was performed with a preload of $0.4 \mathrm{~N}$ and rotation about the long axis of the bone with a speed of $1 \mathrm{~mm} /$ minute until failure. The force applied at failure was recorded and analyzed to calculate the torsional rigidity [newton-millimeters per degree ( $\mathrm{Nmm} /$ degree)], flexibility (degrees), and torque [newton-millimeters (Nmm)]. After failure, the tibiae were visually analyzed to determine the fracture location.

\section{Statistical Analysis}

Data were analyzed using GraphPad Prism software version 5 (GraphPad Software, La Jolla, CA). Unless otherwise specified, data are expressed as means \pm SD. Comparison between groups was performed by use of a one-way analysis of variance test. $P$ values of $\leq 0.05$ were considered significant.

\section{Results}

\section{Time Course of Hematogenous S. aureus Infection in Mice}

C57BL/6 mice were infected intravenously with $10^{6} \mathrm{CFUs}$ of $S$. aureus, and the development of bone infection was evaluated by quantifying the bacterial burden over time in the tibiae of the infected animals. The kinetics of bacterial multiplication were biphasic (Figure 1A). Thus, S. aureus grew progressively in the bones during the first 2 weeks of infection (acute phase). The rate of bacterial replication was considerably reduced after day 15 and stabilized at a constant level from day 30 on, when the infection entered the chronic phase (Figure 1A). The extent of bone infection was dose-dependent (see Supplemental Figure S1 at $h$ ttp://ajp.amjpathol.org).

Although intravenously inoculated $S$. aureus microorganisms were also capable of invading and multiplying in other organs, such as the kidneys, the bacteria were progressively eliminated from these organs, and bacterial clearance was achieved by day 60 of infection (Figure 1A). These observations emphasize the tropism of this strain of $S$. aureus for bone tissue.

Clinical signs of illness were apparent predominantly during the acute phase of the infection; these included weight loss (Figure 1B), swelling, and decreased mobility. In addition, markers of systemic inflammation, such as high levels of serum IL-6 (Figure 1C), were evident only during the very early phase of infection. Between 20\% and $40 \%$ of infected mice died or were euthanized over the course of infection because of weight loss or predefined measures of morbidity. 
A

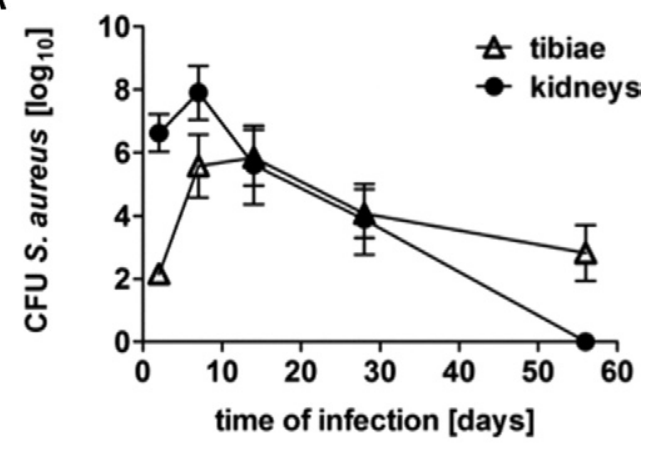

B

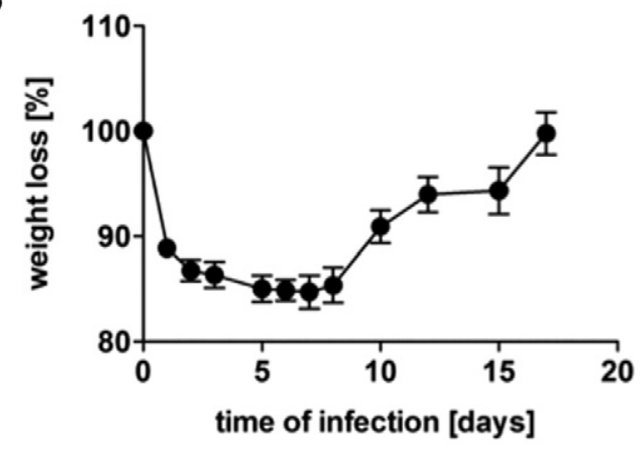

C

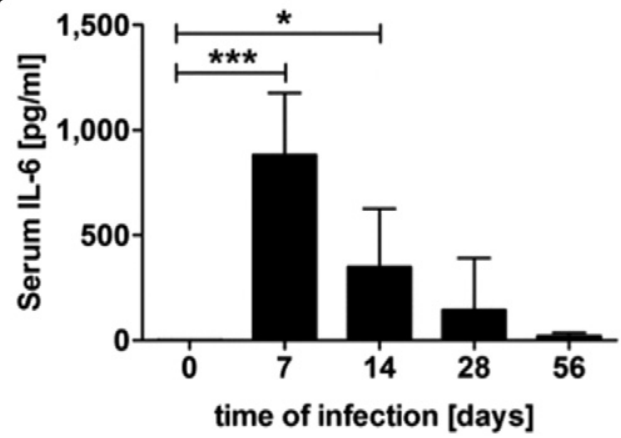

Figure 1. Infection parameters during the course of $S$. aureus osteomyelitis in mice. A: Kinetics of bacterial growth in kidneys and tibiae of mice after intravenous infection with $S$. aureus. B: Percentage weight loss in infected mice over a period of 17 days. C: Levels of IL-6 in serum of infected mice at 7, 14, 28, and 56 days after bacterial inoculation. Data are expressed as means $\pm \mathrm{SD}$ and represent one of three independent experiments with similar results. ${ }^{*} P<0.05,{ }^{* * * *} P<0.001 . n \geq 5$ mice per group.

\section{Imaging Bone Structural Changes by MRI and X-Ray Radiography}

To monitor the development of osteomyelitis during the acute phase of infection, S. aureus-infected mice were subjected to noninvasive MRI and X-ray imaging at intervals over a period of 14 days, and the inflammatory lesions within the infected tibiae were quantified within segmented three-dimensional reconstructions of the magnetic resonance images (Figure 2; Tables 1 and 2). Inflammatory lesions in the tibiae became apparent by MRI in three of the four mice at day 7 of infection. By day 14, all animals exhibited inflammatory lesions in the tibiae, although the size of the lesions and location (right versus left tibia) varied among the mice (Figure 2; Tables 1 and 2).

We also investigated the progression from acute to chronic osteomyelitis of three mice by MRI and X-ray imaging. Repeated MRI within 2 months showed a continuous spreading and expansion of multiple foci of inflammation in the affected tibia during the chronic stage of the infection (Figure 3). The size of inflammation, bone deformation, and bone thickening varied considerably between left and right tibia and among animals (Figure 3; Tables 1 and 2; see also Supplemental Figure S2 at http://ajp.amjpathol.org). In less severe cases, the inflammation was restricted to certain parts of the bone, resulting in only moderate bone thickening (Figure 3; Tables 1 and 2; see also Supplemental Figure S2 at $h$ ttp://ajp. amjpathol.org). In severe cases, the area of inflammation spread through the entire bone, leading to tremendous deformation and up to a fivefold gain in bone volume at day 63 of infection (Figure 3; Tables 1 and 2). Bone abnormalities and deformation started to be evident by X-ray imaging at day 14 of infection (Figure 3; see also Supplemental Figure S2 at http://ajp.amjpathol.org). Furthermore, whole-body X-ray scans taken at days 3 and 63 of infection showed that other bones, such as the humerus, were also affected late in infection (Figure 3).

The magnetic resonance and X-ray findings in the experimental murine model of $S$. aureus osteomyelitis are very similar to those observed in human patients. For comparison with the murine model, we have included the magnetic resonance and X-ray images of the elbow of a 71-year-old man with a 2-month history of bursitis and olecranon osteomyelitis (Figure 4). The patient did not show any signs of systemic inflammation (absence of fever; C-reactive protein at $0.5 \mathrm{mg} / \mathrm{dL}$ ), but he presented with persistent pain, a fistula, and a reddened wound at his elbow. X-ray analysis revealed osteolysis in the olecranon (Figure 4A). The presence of an inflammatory focus was evinced by MRI (Figure 4B). Surgical debridement was performed, and $S$. aureus was cultured from bone specimens, which confirmed the diagnosis of chronic $S$. aureus osteomyelitis.

\section{Histopathological Evaluation of Infected Bones}

The development of osteomyelitis in murine infected bones was further confirmed by histological examination. An image of the medullary cavity of a representative uninfected bone (day 0 ) is shown in Figure 5A. In the murine model, early infection (7 days after inoculation) was characterized by suppurative inflammation with foci of intense bacterial multiplication and necrosis (Figure 5, $\mathrm{B}$ and $\mathrm{C}$ ), similar to human acute osteomyelitis (Figure 5D). All animals $(n=5)$ examined during this early phase of infection exhibited myeloid hyperplasia in the infected bones. The unremitting massive influx of granulocytes replaced the red blood cells, hematopoietic cells, and fat cells in the bone marrow (Figure 5, B and C). During the transition from acute to chronic infection, the massive influx of inflammatory cells decreased, and areas of bone tissue destruction due to the activation of bone-resorbing osteoclasts were observed (Figure 5E). A bone segment 

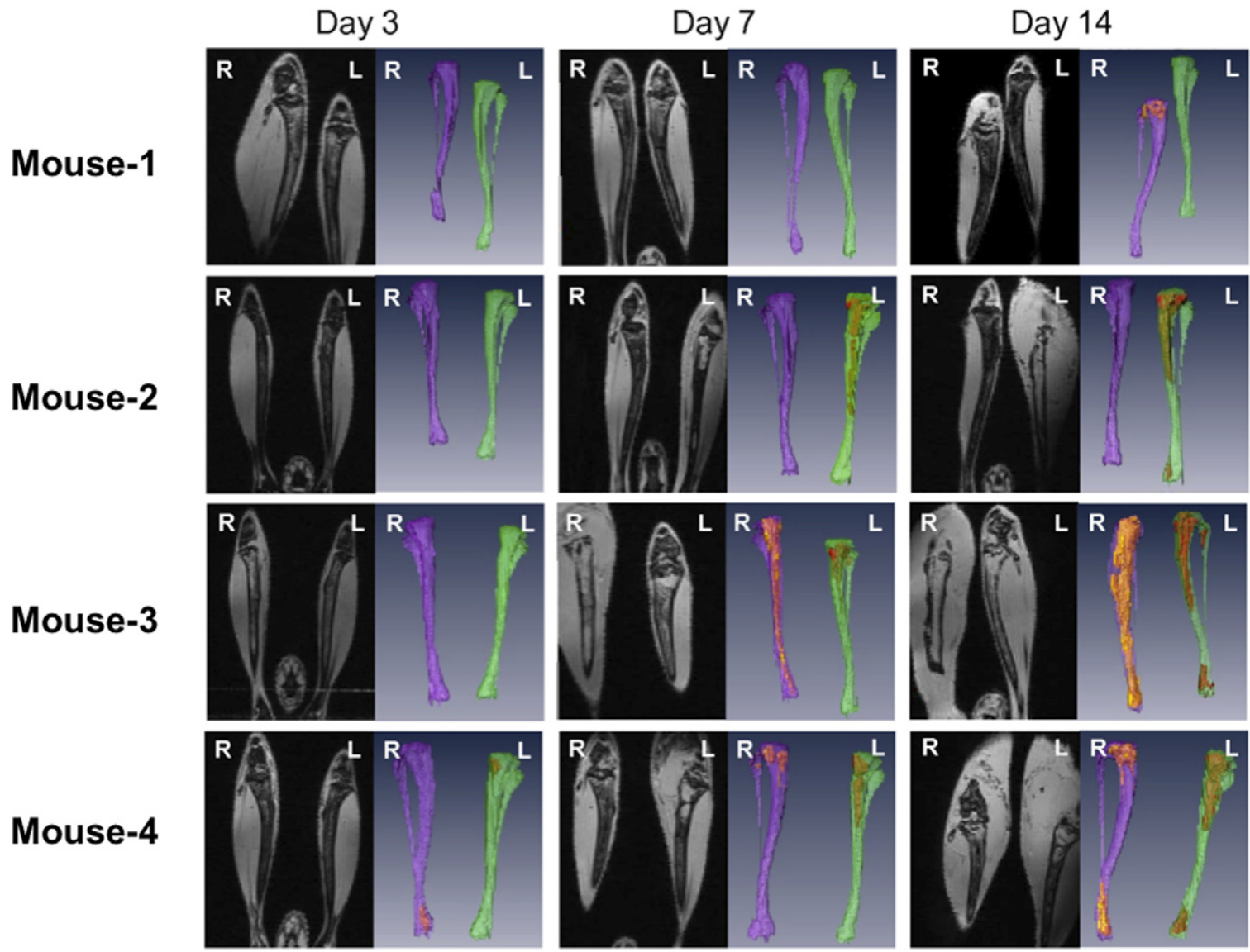

Figure 2. MRI scans and three-dimensional reconstruction of the right (R) and left (L) tibiae as well as inflammatory lesions of four mice during the acute phase of infection (days 3 to 14). The zones of inflammation are well defined and differentiate from the bone structure as hyperintensities in the FLASH images. Inflammatory lesions (zone of inflammation with edema formation) are colored red and brown (left) or orange and pink (right) according to the inflammatory depth in the bones. Noninflamed area (the area of the bone without signs of inflammation) of the right leg is shown in magenta and the noninflamed area of the left leg is shown in green.

exhibiting a ruffled border with the typical resorption lacunae produced by the activity of individual osteoclasts is shown in Figure 5F. Lacunae are typical features of human chronic osteomyelitis (Figure 5G). The activity of osteoclasts often led to the separation of dead bone from the remaining living bone, generating a sequestrum (Figure $5 \mathrm{H}$ ).

Simultaneous with bone destruction was the formation of new bone. We observed reactive bone formation in three of the five mice at day 21 of infection. Reversal lines

Table 1. Volume of Inflammatory Lesions and Noninflamed Areas of the Right Tibia in Three S. aureus-Infected Mice

\begin{tabular}{|c|c|c|c|c|c|c|c|c|c|}
\hline \multirow{3}{*}{$\begin{array}{c}\text { Time of } \\
\text { infection } \\
\text { (days) }\end{array}$} & \multicolumn{9}{|c|}{ Right tibia, volume $\left(\mathrm{mm}^{3}\right)$} \\
\hline & \multicolumn{3}{|c|}{ Lesion } & \multicolumn{3}{|c|}{ Noninflamed } & \multicolumn{3}{|c|}{ Total } \\
\hline & 1 & 2 & 3 & 1 & 2 & 3 & 1 & 2 & 3 \\
\hline 3 & 0 & 0 & 1.3 & 24.3 & 26.4 & 23.5 & 24.3 & 26.4 & 24.8 \\
\hline 7 & 0 & 8.3 & 1.5 & 23.5 & 18.9 & 24.1 & 23.5 & 27.2 & 25.6 \\
\hline 14 & 0 & 18.5 & 2.1 & 25.6 & 23.4 & 24.6 & 25.6 & 41.9 & 26.7 \\
\hline 22 & 1.7 & 28.2 & 1.4 & 23.4 & 45.9 & 24.8 & 25.1 & 74.1 & 26.2 \\
\hline 35 & 1.1 & 29.5 & 5.6 & 24.0 & 43.8 & 21.2 & 25.1 & 73.3 & 26.8 \\
\hline 49 & 0.6 & 34 & 12.5 & 24.6 & 37.6 & 11.1 & 25.2 & 71.6 & 23.6 \\
\hline 63 & 13 & 31 & 13.0 & 13 & 43.8 & 13.0 & 26 & 74.8 & 26.0 \\
\hline
\end{tabular}

indicate newly built bone after bone resorption (Figure $5 F)$. New periosteal bone exhibited the typical irregular structure of woven bone and increased in thickness with the progression toward chronic infection in both murine (Figure 5I) and human (Figure 5J) infected bone. At later time points (28 days after infection), the infected bone area became walled off by fibrous tissue to form a necrotizing pyogranuloma (observed in four of the five mice at 28 days of infection). These pyogranulomas encompass-

Table 2. Volume of Inflammatory Lesions and Noninflamed Areas of the Left Tibia in Three $S$. aureus-Infected Mice

\begin{tabular}{|c|c|c|c|c|c|c|c|c|c|}
\hline \multirow{3}{*}{$\begin{array}{c}\text { Time of } \\
\text { infection } \\
\text { (days) }\end{array}$} & \multicolumn{9}{|c|}{ Left tibia, volume $\left(\mathrm{mm}^{3}\right)$} \\
\hline & \multicolumn{3}{|c|}{ Lesion } & \multicolumn{3}{|c|}{ Noninflamed } & \multicolumn{3}{|c|}{ Total } \\
\hline & 1 & 2 & 3 & 1 & 2 & 3 & 1 & 2 & 3 \\
\hline 3 & 0 & 0 & 2.3 & 21.5 & 28.3 & 23.7 & 21.5 & 28.3 & 26.0 \\
\hline 7 & 0 & 2.4 & 7.2 & 25.6 & 24.9 & 19.4 & 25.6 & 27.3 & 26.6 \\
\hline 14 & 0 & 0 & 17.1 & 28.2 & 30.4 & 20.9 & 28.2 & 30.4 & 38.0 \\
\hline 22 & 2.8 & 0 & 25.4 & 26.2 & 31.3 & 47.3 & 29.0 & 31.3 & 72.7 \\
\hline 35 & 4.5 & 0 & 31.4 & 25.2 & 30.5 & 86.0 & 29.7 & 30.5 & 117.4 \\
\hline 49 & 5.7 & 0 & 49.0 & 23.1 & 28.5 & 79.5 & 28.8 & 28.5 & 128.5 \\
\hline 63 & 7.2 & 0 & 44.0 & 21.8 & 30.7 & 83 & 29.1 & 30.7 & 127.0 \\
\hline
\end{tabular}




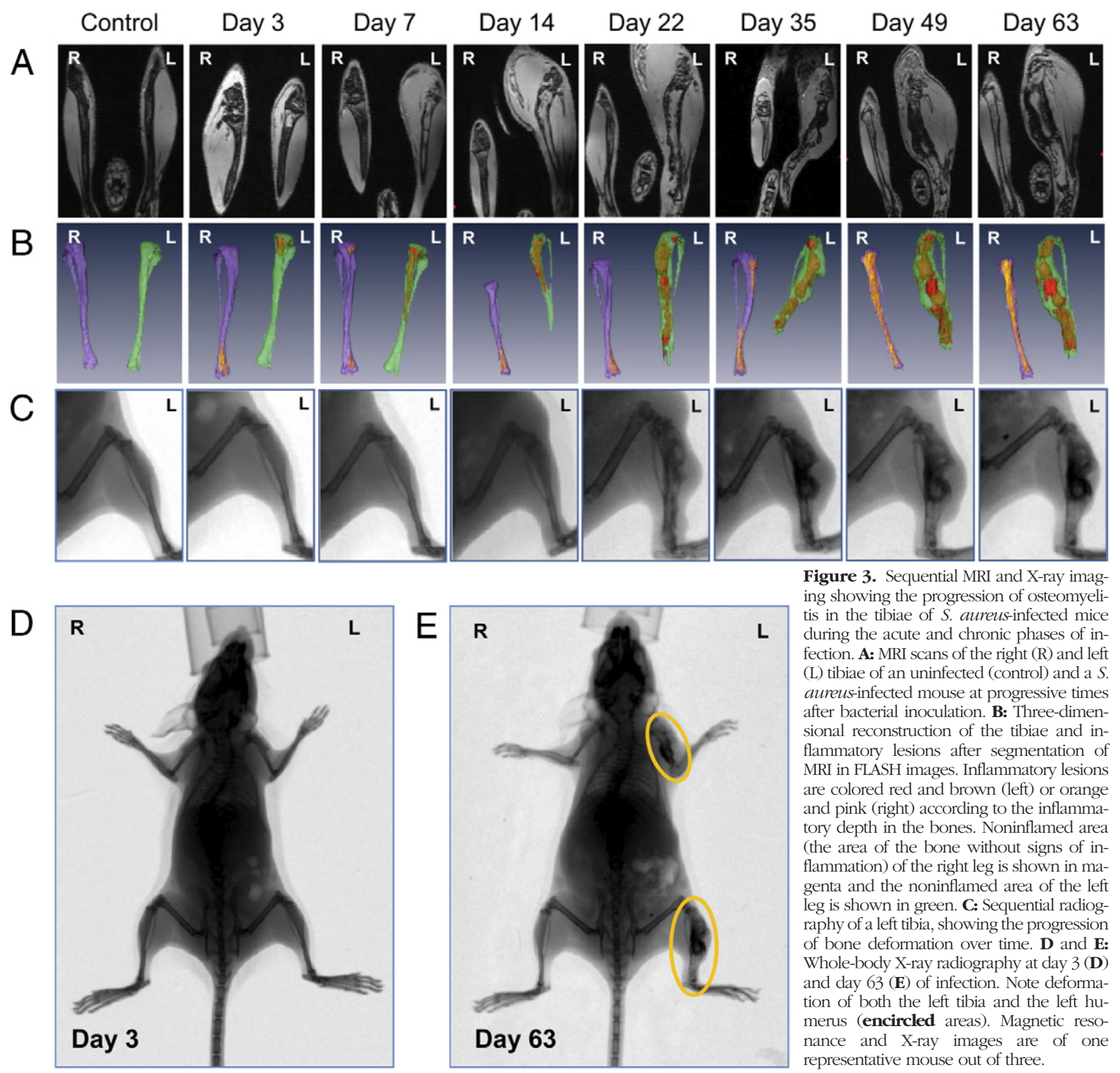

ing necrotic centers surrounded by a layer of vacuolar macrophages, lymphocytes, and granulocytes and an outer layer of fibroblasts are typical features of chronic osteomyelitis (Figure 5K). The combined activity of bone resorption and new bone formation continued during the chronic phase of infection, and extensive bone remodeling was observed at day 56 after bacterial inoculation. Trabeculae appeared as irregular and partially resorbed structures (Figure 5L).

\section{Biomechanical Testing}

Biomechanical testing provides estimates of strength and toughness, which are directly related to the risk of fracture. Uninfected murine bones showed a mean torsional rigidity of $0.97 \pm 0.05 \mathrm{Nmm} /$ degree, a mean flexibility of $15.78 \pm 1.86$ degrees, and a mean torque of $10.71 \pm$ 1.17 Nmm and exhibited one clear fracture (Figure 6). In contrast, infected tibiae (2 months after infection) displayed multiple incomplete fractures and increasing tor- sional rigidity, which strongly depended on the volume of the bone deformation. The flexibility of infected tibiae decreased by approximately $30 \%$, indicating an increased risk of fracture (Figure 6). Although in the control group the fracture occurred in the middle of the shaft, in the infected group the tibiae fractured in the metaphysis (data not shown).

\section{TEM Localization of S. aureus Microorganisms within the Infected Bone}

We next used TEM to determine the precise location of $S$. aureus microorganisms within the infected bones during the acute and chronic phases of infection. At day 10 of infection, S. aureus was found within the nonmineralized collagen matrix of the bone as isolated microorganisms or forming microcolonies (Figure 7, A and B). At day 28, S. aureus was also found internalized within neutrophils (Figure 7, C and D). Some neutrophils harbored a high 

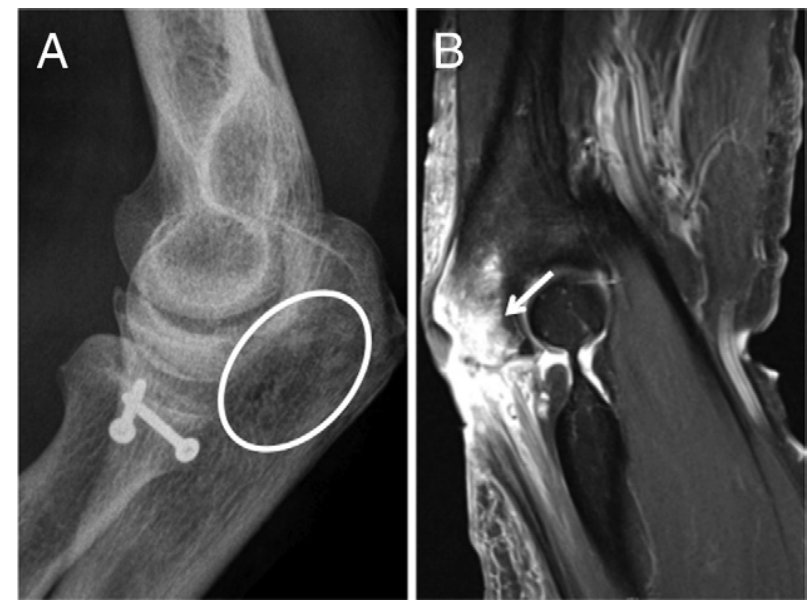

Figure 4. Imaging of a patient with chronic $S$. aureus osteomyelitis in the humerus. A: X-radiograph of the humerus of a 71-year-old man affected by olecranon osteomyelitis for 2 months. The bone area undergoing osteolysis is circled. B: MRI reveals an inflammatory focus in the olecranon (arrow).

number of phagocytized bacteria (Figure 7D), which were released into the extracellular milieu on host cell death (Figure 7E). At day 56 of infection, S. aureus was found mainly in microcolonies containing a mixture of both actively growing and microorganisms (Figure 7, F and $\mathrm{G}$ ).

As in the murine model, morphologically intact and actively dividing $S$. aureus microorganisms could be de-

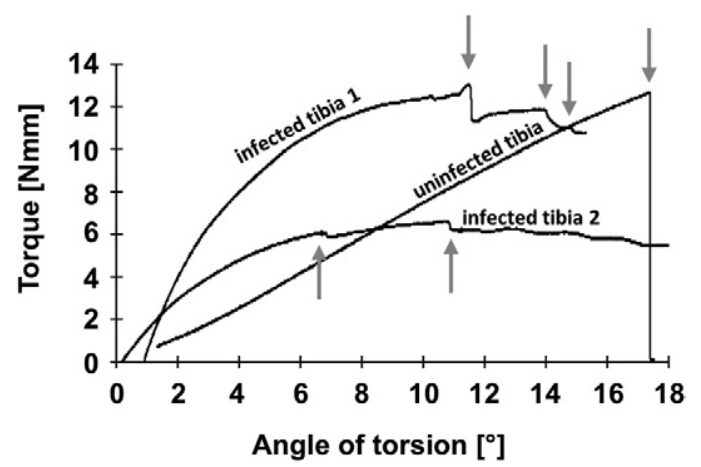

Figure 6. Biomechanical evaluation of long-term $S$. aureus-infected bones. Representative torque-twist curves of one uninfected and two S. aureusinfected mouse tibiae taken at day 63 after bacterial inoculation. Infected tibiae were derived from two different mice. Stress fractures are indicated by arrows

tected by TEM in infected bone tissue surgically removed from a patient affected by staphylococcal osteomyelitis (Figure 7, $\mathrm{H}$ and $\mathrm{I}$ ).

\section{Discussion}

Osteomyelitis caused by $S$. aureus is a severe bone infection and is frequently associated with treatment failure and poor outcome. Although diverse animal models of osteomyelitis have been established, including rabbits, dogs, chickens, rats, and mice, ${ }^{20-22}$ progress in under-

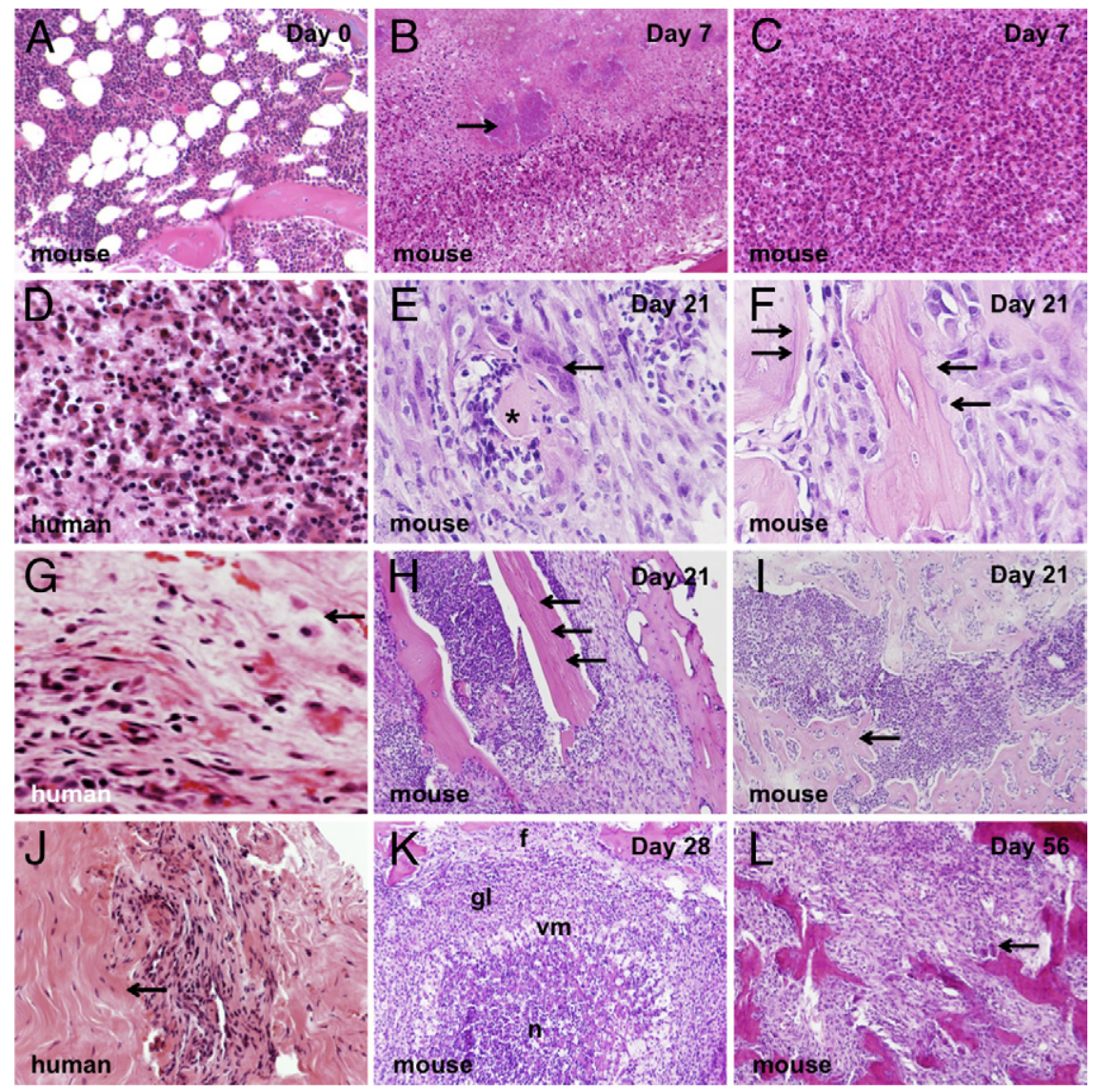

Figure 5. Histological evaluation of mouse and human osteomyelitis during the acute and chronic disease stages (H\&E stain). A: Section of the tibia of an uninfected mouse. $\mathbf{B}$ and $\mathbf{C}$ : Sections of the tibia of a $S$. aureus-infected mouse at day 7 of infection showing bacterial microcolonies $(\mathbf{B}$, arrow) and infiltrating granulocytes $(\mathbf{C})$. D: Section of a bone specimen taken from a patient with acute osteomyelitis. Note the massive infiltration of inflammatory cells. $\mathbf{E}$ and $\mathbf{F}$ : Sections of a tibia of a $S$. aureus-infected mouse at day 21 of infection showing a bone sequestrum $(\mathbf{E}$, asterisk) with an attached active osteoclast (E, arrow), lacunae (F, right arrows), and reversal line (F, left arrows). G: Section of a bone specimen taken from a patient with chronic osteomyelitis. Note lacuna due to bone resorption (arrow). $\mathbf{H}$ and I: Sections of a tibia of a $S$. aureus-infected mouse at day 21 of infection showing a bone sequestrum due to infraction $(\mathbf{H}$, arrows) and woven bone $(\mathbf{I}$, arrow). $\mathbf{J}$ : Section of a bone specimen taken from a patient with chronic osteomyelitis showing woven bone (arrow). K: Abscess in the tibia of a S. aureus infected mouse at day 28 of infection comprising a necrotic center ( $n$ ), a rim of vacuolated macrophages $(\mathrm{vm})$, a border of surrounding granulocytes and lymphocytes (gl), and fibrous tissue (f). L: Sections of the tibia of a S. aureus-infected mouse at day 56 of infection showing partially resorbed trabeculae with attached osteoclasts (arrow). Original magnification: $\times 10(\mathbf{C}, \mathbf{H}, \mathbf{I}$, $\mathbf{K}, \mathbf{L}) ; \times 20(\mathbf{A}, \mathbf{B}, \mathbf{J}) ; \times 40(\mathbf{D}, \mathbf{E}, \mathbf{F}, \mathbf{G})$. 


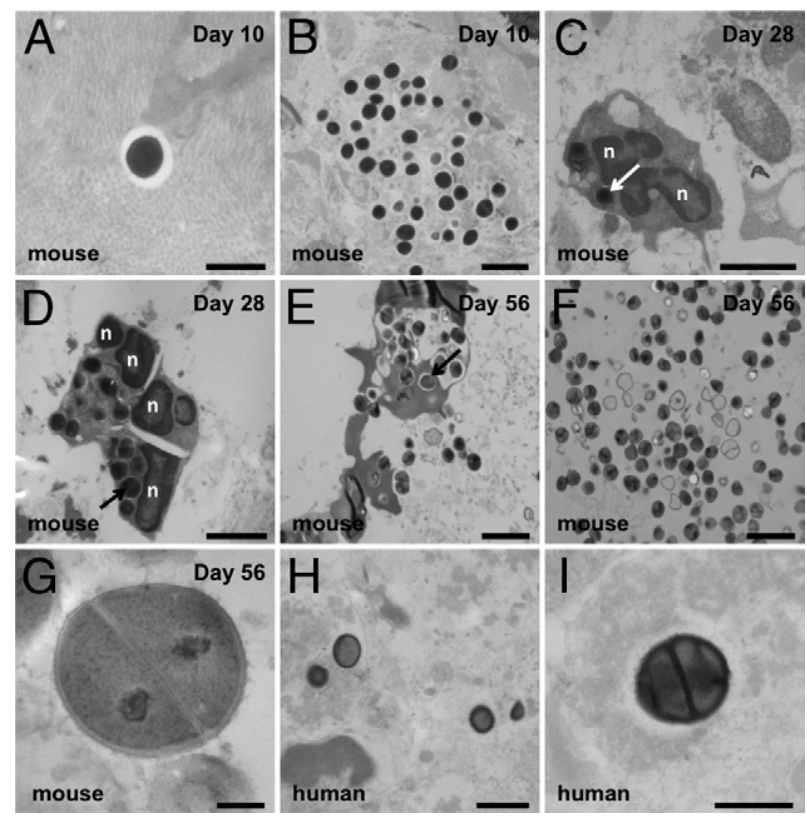

Figure 7. TEM examination of $S$. aureus microorganisms in murine and human infected bones. A and B: At day 10 of infection, S. aureus within murine bones was found embedded in the collagen matrix as isolated microorganisms (A) or in microcolonies (B). C and D: At day 28 of infection, $S$ aureus (arrows) was found within neutrophils (n). E: Staphylococci are released into the extracellular milieu by dying neutrophils. F: At day 56 of infection, large bacterial colonies were found, composed of a mix of deac and actively dividing microorganisms. G: Actively dividing S. aureus microorganisms in murine bones at day 56 of infection. H: $S$. aureus microorganisms in chronically infected human bone. I: Actively dividing $S$. aureus microorganisms in chronically infected human bone. Scale bar: $10 \mu \mathrm{m}(\mathbf{F}) ; 2$ $\mu \mathrm{m}(\mathbf{B}-\mathbf{E}) ; 1 \mu \mathrm{m}(\mathbf{A}$ and $\mathbf{H}) ; 500 \mathrm{~nm}(\mathbf{I}) ; 200 \mathrm{~nm}(\mathbf{G})$.

standing the pathophysiology of this problematic infection has been suboptimal because models are lacking that adequately reflect the full spectrum of the human disease, including both acute and chronic phases. To overcome this limitation, we developed a novel murine model of osteomyelitis in which bone infection is induced after injection of $S$. aureus microorganisms directly into the bloodstream. This murine model recapitulates important aspects of the acute and chronic stages of osteomyelitis in humans as demonstrated by histopathology, MRI, X-ray imaging, and electron microscopic examination.

Osteomyelitis can be subdivided into two distinct clinical phases: acute and chronic disease. The murine model of osteomyelitis developed in the present study reliably mimics the clinical features of these two stages. Thus, the acute phase of infection (the first 2 weeks) is highly symptomatic and is characterized by progressive bacterial growth in bones and high levels of systemic inflammation as evinced by weight loss and elevated serum levels of IL-6. X-ray findings are typically normal during this initial phase of infection. During the chronic stage (1 month after bacterial inoculation), inflammation is locally restricted to the infected bones, and changes in the bone structure become or are already evident by X-ray imaging (osteolysis, deformation) and MRI (hyperintense areas of tissue edema). The infected bones undergo tremendous deformation induced by destruction and remodeling processes. Biomechanical measurements indicate that flexibility decreases and torsional ri- gidity increases in infected bones, resulting in increased risk for fractures. Bone destruction, deformation, and fracture are common and dreaded complications of the various types of osteomyelitis. Children with hematogenous $S$. aureus osteomyelitis are at an increased risk for bone destruction and pathological fractures, particularly if subperiosteal abscesses are present and if therapy starts late. ${ }^{8,23}$

Histopathological evaluation of infected bones revealed a massive infiltration of inflammatory cells during the acute phase of the infection. As in the human disease, bone resorption started to be apparent when the infection entered in the chronic phase (3 weeks after bacterial inoculation) (Figure 5F). We could also detect formation of bone sequestra in this murine model of $S$. aureus osteomyelitis (Figure $5 \mathrm{H}$ ). Sequestra are fragments of necrotic bone. These fragments are avascular and often harbor live bacteria, which are generally refractory to antibiotic treatment. As a consequence, the infected sequestrum can remain a focus of recurrent infection, and surgical intervention is the most effective way to sterilize the infection. ${ }^{24}$ Alternatively, local delivery of a high concentration of antibiotics at the site of infection has been successfully applied for the treatment of osteomyelitis. ${ }^{25}$ However, this treatment procedure requires an antibiotic delivery system, and subsequently a second intervention for removal of the antibiotic delivery device. To overcome this inconvenience, new delivery methods are being developed, such as the use of antibiotic-impregnated biodegradable beads. ${ }^{26,27}$ In this regard, the murine model of osteomyelitis described here provides a suitable tool for testing new antimicrobial strategies or more manageable antibiotic delivery systems. Although both the volume of infectious lesions and the extent of bone deformation vary among individual animals, quantification of treatment effects is feasible. Using noninvasive imaging methods, quantitative data can be obtained before treatment is started, and may serve as baseline for each individual animal.

An additional advantage of our murine model of hematogenous osteomyelitis is that, in contrast to most published osteomyelitis models in which staphylococci are directly placed into the bones after traumatic injury, ${ }^{21,22,28}$ in our model the bacteria are seeded into the bones after spreading via the bloodstream. This mimics the natural route of infection in hematogenous osteomyelitis and can facilitate the identification of bacterial factors involved in bone tropism. This is important, because the potential virulence factors used by staphylococci to infect the bones remains a matter of debate. ${ }^{29-31}$

Staphylococci have evolved specific pathogenic mechanisms that contribute to bacterial persistence and development of chronic infection. Despite previous evidence showing that a number of staphylococcal genes are relevant in persistent infection, ${ }^{32}$ there are enormous gaps in our understanding of the location and physiological status of persistent bacteria during in vivo infection. The murine model developed in the present study can serve as platform to characterize the temporally ordered series of transcriptional responses of $S$. aureus microorganisms that affect stage-specific bacterial adaptation to 
the bone tissue during acute and chronic infection. These findings could provide the foundation for the development of new therapeutic strategies for patients with chronic osteomyelitis.

In summary, we have described a novel murine model of $S$. aureus hematogenous osteomyelitis that reflects the full spectrum of the human disease. This model provides an important platform for microbiological and immunological studies on chronic osteomyelitis and for the evaluation of novel approaches for diagnostics and treatment.

\section{References}

1. Wright JA, Nair SP: Interaction of staphylococci with bone. Int J Med Microbiol 2010, 300:193-204

2. Lew DP, Waldvogel FA: Osteomyelitis. Lancet 2004, 364:369-379

3. Chambers HF: Community-associated MRSA-resistance and virulence converge. N Engl J Med 2005, 352:1485-1487

4. Plouin-Gaudon I, Clement S, Huggler E, Chaponnier C, François P, Lew D, Schrenzel J, Vaudaux P, Lacroix JS: Intracellular residency is frequently associated with recurrent Staphylococcus aureus rhinosinusitis. Rhinology 2006, 44:249-254

5. Sheehy SH, Atkins BA, Bejon P, Byren I, Wyllie D, Athanasou NA, Berendt AR, McNally MA: The microbiology of chronic osteomyelitis: prevalence of resistance to common empirical anti-microbial regimens. J Infect 2010, 60:338-343

6. Foster TJ: Immune evasion by staphylococci. Nat Rev Microbiol 2005, 3:948-958

7. Lowy FD: Staphylococcus aureus infections. N Engl J Med 1998, 339:520-532

8. Belthur MV, Birchansky SB, Verdugo AA, Mason EO Jr, Hulten KG, Kaplan SL, Smith EO, Phillips WA, Weinberg J: Pathologic fractures in children with acute Staphylococcus aureus osteomyelitis. J bone Joint Surg Am 2012, 94:34-42

9. Jones HW, Beckles VL, Akinola B, Stevenson AJ, Harrison WJ: Chronic haematogenous osteomyelitis in children: an unsolved problem. J bone Joint Surg Br 2011, 93:1005-1010

10. Lawrence SM, Wraight PR, Campbell DA, Colman PG: Assessment and management of inpatients with acute diabetes-related foot complications: room for improvement. Intern Med J 2004, 34:229_ 233

11. Powlson AS, Coll AP: The treatment of diabetic foot infections. J Antimicrob Chemother 2010, 65 Suppl 3:iii3-iii9

12. Rao N, Ziran BH, Lipsky BA: Treating osteomyelitis: antibiotics and surgery. Plast Reconstr Surg 2011, 127 Suppl 1:177S-187S

13. Conrad DA: Acute hematogenous osteomyelitis. Pediatr Rev 2010, $31: 464-471$

14. Carek PJ, Dickerson LM, Sack JL: Diagnosis and management of osteomyelitis [Erratum appeared in Am Fam Physician 2002, 65: 1751]. Am Fam Physician 2001, 63:2413-2420
15. Ciampolini J, Harding KG: Pathophysiology of chronic bacterial osteomyelitis. Why do antibiotics fail so often? Postgrad Med J 2000 , 76:479-483

16. Korovessis $P$, Fortis AP, Spastris $P$, Droutsas P: Acute osteomyelitis of the patella 50 years after a knee fusion for septic arthritis. A case report. Clin Orthop Relat Res 1991, 272:205-207

17. Gallie WE: First recurrence of osteomyelitis eighty years after infection. J Bone Joint Surg Br 1951, 33B:110-111

18. Proctor RA, Christman G, Mosher DF: Fibronectin-induced agglutination of Staphylococcus aureus correlates with invasiveness. J Lab Clin Med 1984, 104:455-469

19. Spurr AR: A low-viscosity epoxy resin embedding medium for electron microscopy. J Ultrastruct Res 1969, 26:31-43

20. Patel M, Rojavin Y, Jamali AA, Wasielewski SJ, Salgado CJ: Animal models for the study of osteomyelitis. Semin Plast Surg 2009, 23:148154

21. Funao H, Ishii K, Nagai S, Sasaki A, Hoshikawa T, Aizawa M, Okada Y, Chiba K, Koyasu S, Toyama Y, Matsumoto M: Establishment of a real-time, quantitative, and reproducible mouse model of Staphylococcus osteomyelitis using bioluminescence imaging. Infect Immun 2012, 80:733-741

22. Li D, Gromov K, Søballe K, Puzas JE, O'Keefe RJ, Awad H, Drissi H, Schwarz EM: Quantitative mouse model of implant-associated osteomyelitis and the kinetics of microbial growth, osteolysis, and humoral immunity. J Orthop Res 2008, 26:96-105

23. Shetty AK, Kumar A: Osteomyelitis in adolescents. Adolesc Med State Art Rev 2007, 18:79-94, ix

24. Parsons B, Strauss E: Surgical management of chronic osteomyelitis. Am J Surg 2004, 188(1A Suppl):57-66

25. Gogia JS, Meehan JP, Di Cesare PE, Jamali AA: Local antibiotic therapy in osteomyelitis. Semin Plast Surg 2009, 23:100-107

26. Liu SJ, Wen-Neng Ueng S, Lin SS, Chan EC: In vivo release of vancomycin from biodegradable beads. J Biomed Mater Res A 2002 63:807-813

27. Neut D, Kluin OS, Crielaard BJ, van der Mei HC, Busscher HJ, Grijpma DW: A biodegradable antibiotic delivery system based on poly-(trimethylene carbonate) for the treatment of osteomyelitis. Acta Orthop 2009, 80:514-519

28. Johansson A, Flock JI, Svensson O: Collagen and fibronectin binding in experimental staphylococcal osteomyelitis. Clin Orthop Relat Res 2001, 382:241-246

29. Elasri MO, Thomas JR, Skinner RA, Blevins JS, Beenken KE, Nelson CL, Smeltzer MS: Staphylococcus aureus collagen adhesin contributes to the pathogenesis of osteomyelitis. Bone 2002, 30:275-280

30. Darouiche RO, Landon GC, Patti JM, Nguyen LL, Fernau RC, McDevitt D, Greene C, Foster T, Klima M: Role of Staphylococcus aureus surface adhesins in orthopaedic device infections: are results model-dependent? J Med Microbiol 1997, 46:75-79

31. Tung H, Guss B, Hellman U, Persson L, Rubin K, Rydén C: A bone sialoprotein-binding protein from Staphylococcus aureus: a member of the staphylococcal Sdr family. Biochem J 2000, 345:611-619

32. Tuchscherr L, Medina E, Hussain M, Völker W, Heitmann V, Niemann S, Holzinger D, Roth J, Proctor RA, Becker K, Peters G, Löffler B: Staphylococcus aureus phenotype switching: an effective bacterial strategy to escape host immune response and establish a chronic infection. EMBO Mol Med 2011, 3:129-141 